\title{
HAND INJURY IN BOXING
}

\author{
A. McDOUGALL, F.R.C.S., F.R.F.P.S.
}

Victoria Infirmary, Glasgow.

In a contest the boxer uses his hands to attack and he scores points when he punches his opponent on the target area (the front of the body above the belt) using the knuckle part of the closed fist. When the punch is delivered the hand must be closed and he must not slap or strike his opponent with the side of the hand, the forearm, or the elbow, but he can use these parts of the upper limbs to defend himself. Depending on the nature of the contest boxing gloves weighing eight or six ounces are used and the hands may be bandaged, a regulation gauze or light crepe bandage nine feet long must be used.

Providing the boxer adheres to the rules and punches with the hand in the correct position it is seldom that the hand is injured. Most of the hand injuries in boxing are sustained during training sessions when the heavy punch bags are being used, and the hands are not properly supported with well fitting training mitts.

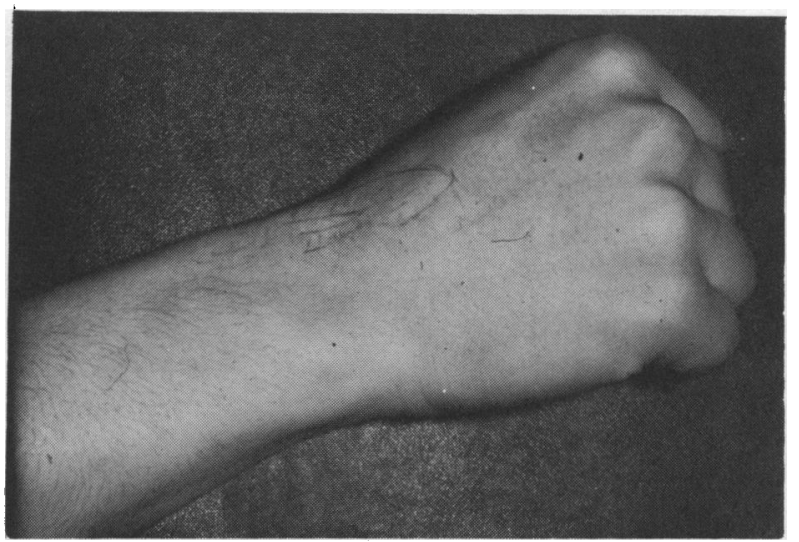

Fig. 1. A closed fist showing prominence of head of middle metacarpal.

Also shown are the extensors of the carpus (marked) at their insertion, a common site for swelling to occur.

When the hand is closed to make a fist (Fig. 1) the head of the middle metacarpal projects beyond the rest and is liable to be traumatised; an enlarged bursa forms over the joint, the capsular tissues swell and an effusion forms in the joint. Once the bursa is present the hand must be rested, analgesic ointments can be applied, hot hand baths prescribed and all blows on the knuckle must be avoided. Cortisone injections are used and they may shorten the disability period, but I have found that if pain is relieved at an early stage the boxer will return too soon to his previous routine with the chance of early recurrence. Once upon a time boxers tried to toughen the skin of the hands by the use of brine, or alum hand baths, but I am not convinced that these were of any value; a much more rational approach is proper bandaging of the hands, proper maintenance of gloves and mitts and the delivery of the punch in the correct manner.

Soft tissue swelling may occur at the bases of the metacarpals of the index and middle fingers; these swellings are also found in golfers, cricket and tennis players and other sportsmen who use the hand to grip tightly an object which is subjected to repeated blows. (Fig. 1) The tendons of the long and short extensor of the carpus are attached to these metacarpals and help to stabilise the wrist at the moment of impact. Over a period a soft tissue swelling appears and this is followed by a bony outgrowth on the dorsum of the hand.

Treatment is along general lines as for the metacarpal bursa, if the bone reaction is excessive it can be removed by operation, the attachment of the tendons must be carefully preserved.

Injury to the interphalangeal joints is not common because they are very stable in a closed fist, but occasionally a mallet finger lesion may occur, when the tip of an improperly closed finger strikes an opponent. The deformity is due to an extensor tendon being pulled forcibly from its attachment to the base of the distal phalanx, a small fragment of bone may be pulled off with it. (Fig. 2)

This apparently inoccuous injury can be very painful with marked swelling around the joint of such a nature as to suggest an acute inflammatory lesion. Many forms of splintage and plaster fixation have been advocated all of which immobilise the whole finger and interfere with the use of the hand. For some time now I have treated this injury by applying a small felt pad to the pulp of the finger and one to the dorsum of the middle phalanx just proximal to the swelling, holding the terminal phalanx in full extension one inch micropore is applied in four layers from the proximal interphalangeal joint to the tip of the finger. This is removed and reapplied weekly for six weeks, keeping the terminal joint straight. At the end of this period the patient is instructed to reapply the pads and strapping himself for a further month; at the end of this time the finger remains straight and the pain 


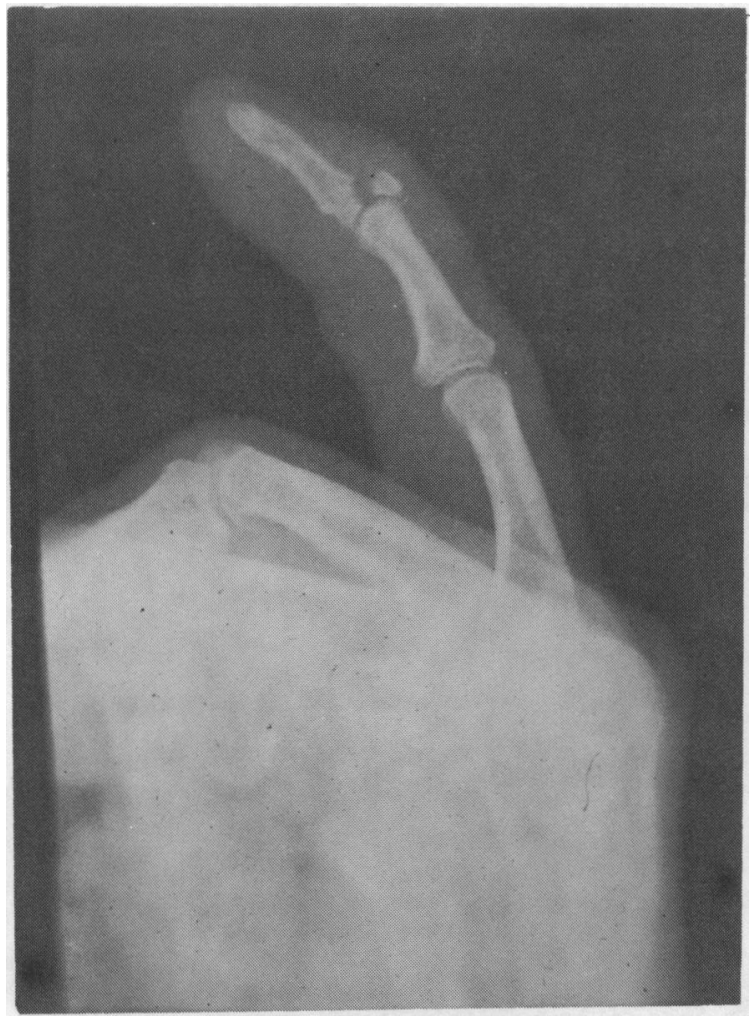

Fig. 2. Mallet finger, the extensor tendon has avulsed its bony insertion.

and swelling disappear. Using this technique most people can continue at work.

Fractures of the phalanges differ in no way from similar injuries in other sports and treatment is standard. It should be emphasised that any angular deformity present must be corrected and the proper alignment of the finger obtained otherwise the function of the hand

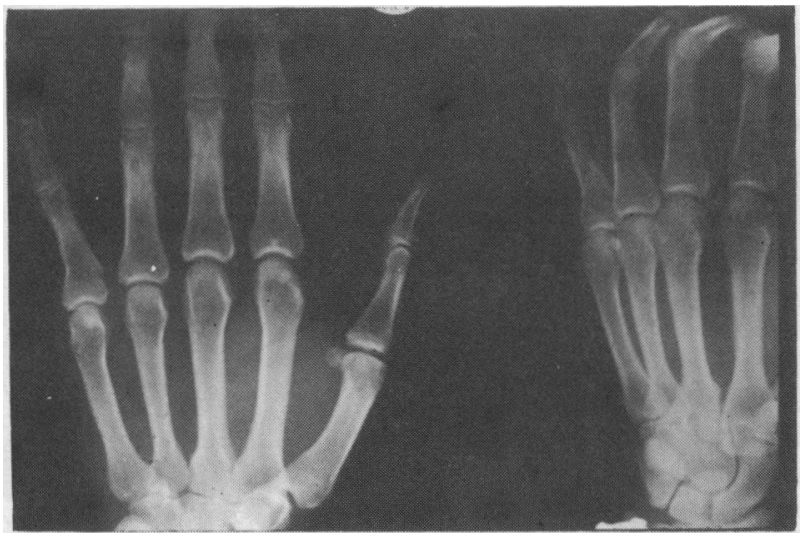

Fig. 3. Fracture of the base of the proximal phalanx of small finger, with ulnar deviation. may be impaired and the hand may not close completely. (Fig. $3 \& 4$ )

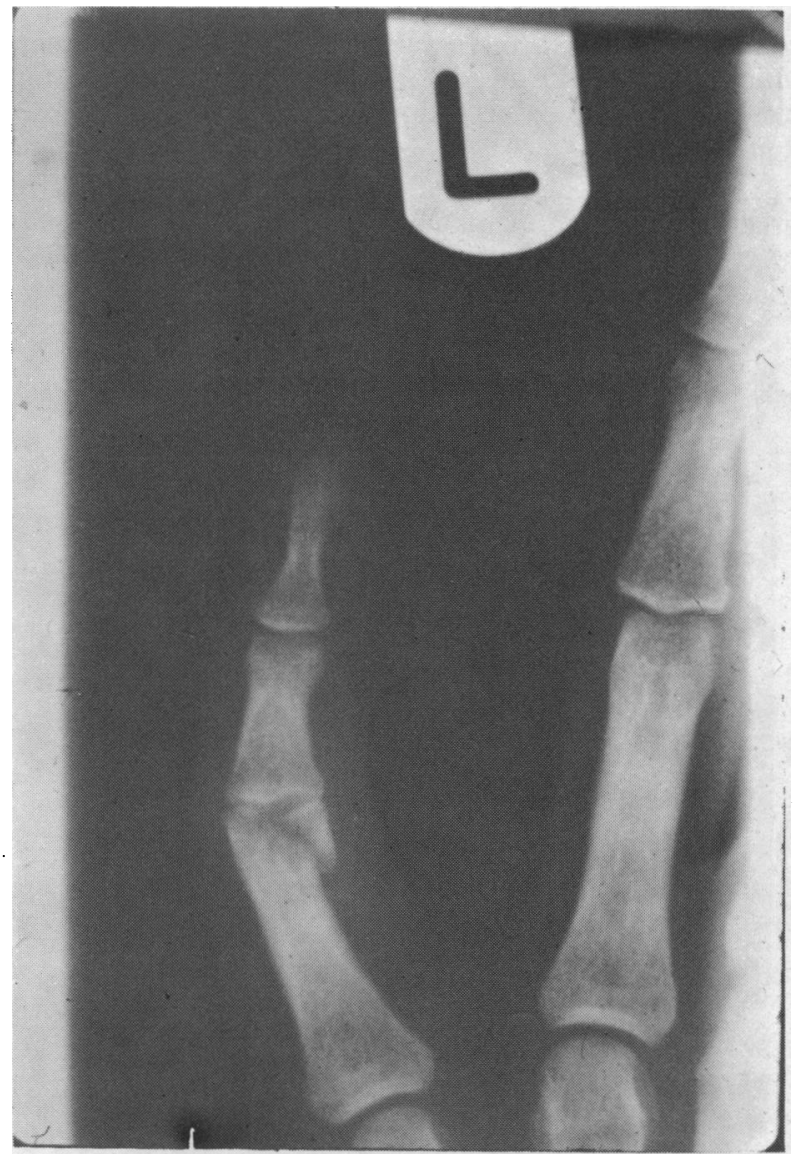

Fig. 4. Fracture of head of proximal phalanx of small finger with radial deviation.

When the hand is closed the fingers point to the tubercle of the scarhoid at the base of the thenar eminence. The majority of fractures of the phalanges can be adequately treated by gently closing the fist over a soft sorbo rubber pad and bandaging it in this position. This gives the functional "tumbler grasping" position. Gentle movements are encouraged within the limits of the bandaging, which is reapplied weekly, allowing more freedom; by the end of the third week the hand can be left free and the patient encouraged to mobilise the fingers in hot soapy water hand baths. As a general rule fingers should not be immobilised for more than three weeks, if joint stiffness is to be avoided.

Injuries to the metacarpals are more common, this is not surprising as the force of a punch is transmitted along the metacarpal shafts to the wrist and forearm. It could be said that these bones are broken not so much in boxing as in fighting, including the Friday night variety. 
The metacarpal of the small finger and to a lesser degree that of the index are vulnerable when a blow is struck when the hand is not closed properly. The fracture through the neck of the fifth metacarpal is known as a boxer's fracture; in this injury the small distal fragment which includes the head and neck of the bone is tilted forward into the palm, following the force of the blow. (Fig. 5)

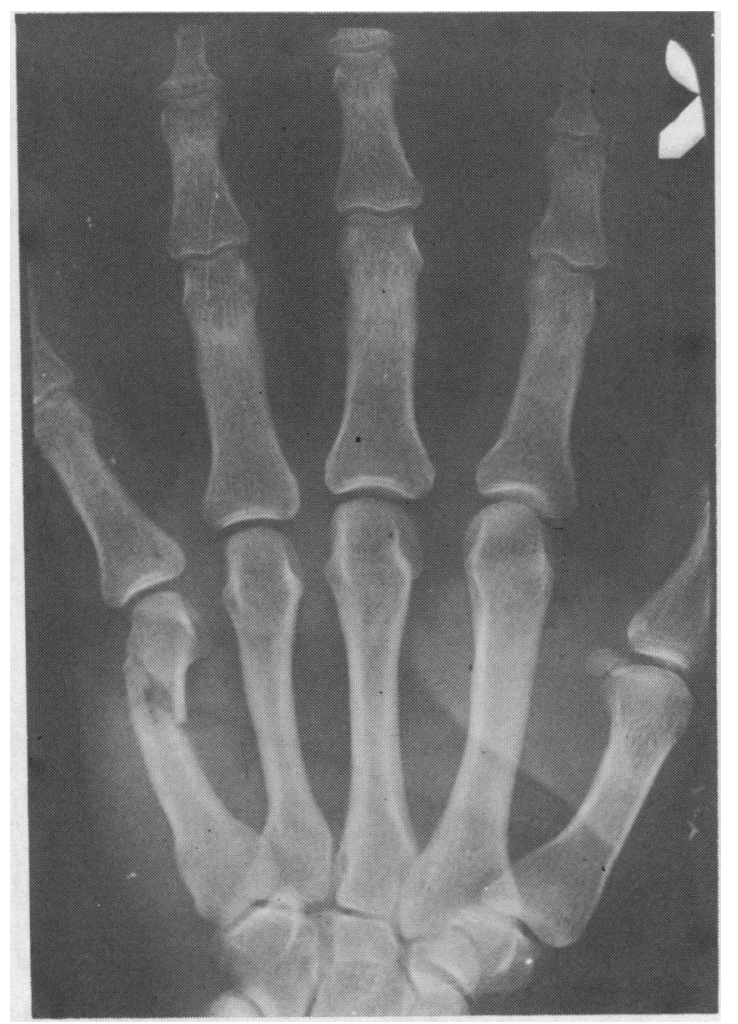

Fig. 5. Fracture of the neck of the fifth metacarpal (Boxer's fracture).

The displacement can be corrected by flexing the metacarpo-phalangeal joint to a right angle, pressure can now be applied along the proximal phalanx to correct the forward tilt of the metacarpal head. This can be done in most cases using local anaesthesia; a firm sorbo pad is placed in the palm, the fingers closed over it and a plaster cast is applied over the dorsum of the hand to cover the small and ring fingers. The plaster is retained for three weeks after which a crepe bandage is bound over the fingers in the same position and gentle mobilising exercises are commenced. Eight weeks should be allowed for union, following which training is progressed as indicated.

The thumb is the digit most liable to injury because of its exposed position, resting on top of the other fingers when the hand is closed. If the boxer punches incorrectly the thumb may catch his opponent's head and be forced radially tearing the ulnar collateral ligament of the metacarpo-phalangeal joint. Occasionally it may be dislocated but this is not so common. (Fig 6)

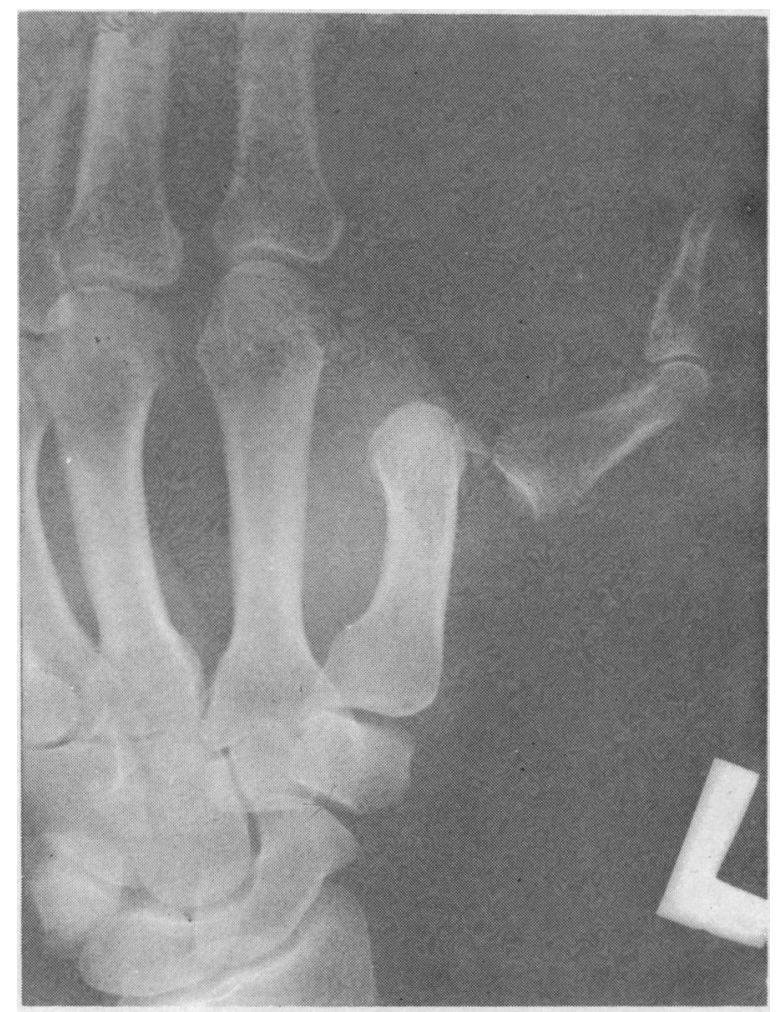

Fig. 6. Dislocation of thumb.

The same injury to the ulnar (medial) collateral ligament of the thumb may be sustained by other athletes when a club or racquet is torn forcibly from the hand. This can be a serious injury and should not be treated lightly. The ulnar collateral ligament stabilises this joint when an object is grasped firmly in the hand, its rupture gives rise to a disabling instability at the joint which interferes with the proper grip of the hand and this may put paid to the career of an athlete not only in his sport but also at his work if it entails the use of hand tools.

In the normal anatomy of the hand the ulnar collateral ligament is covered by the tendinous insertion of the adductor muscle, when the thumb is forcibly abducted the ligament ruptures and may then come to lie on the adductor which separates it from its point of insertion in the phalanx. If this happens the normal anatomy of the joint cannot be restored and the joint remains unstable. (Fig. 7)

When complete rupture of the ligament is indicated by marked instability operative repair should be carried 


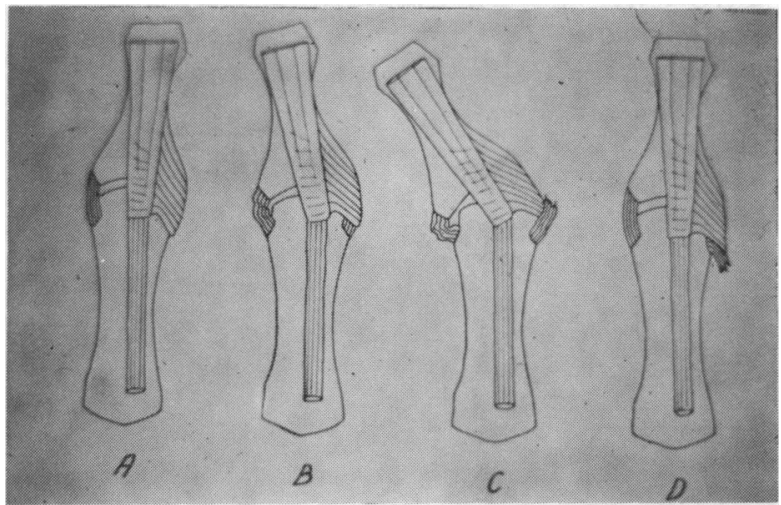

Fig. 7. After Steiner B. British Journal of Bone and Joint Surgery 1962.

A. Normal anatomy of metacarpophalangeal joint of thumb, showing radial collateral ligament of joint, the ulnar collateral ligament is concealed by the tendon of the adductor muscle.

$B$. Abduction at metacarpophalangeal joint.

C. Further abduction with rupture of the ulnar collateral ligament.

D. When the proximal phalanx returns to its original position the torn ulnar collateral ligament is displaced by the adductor tendon.

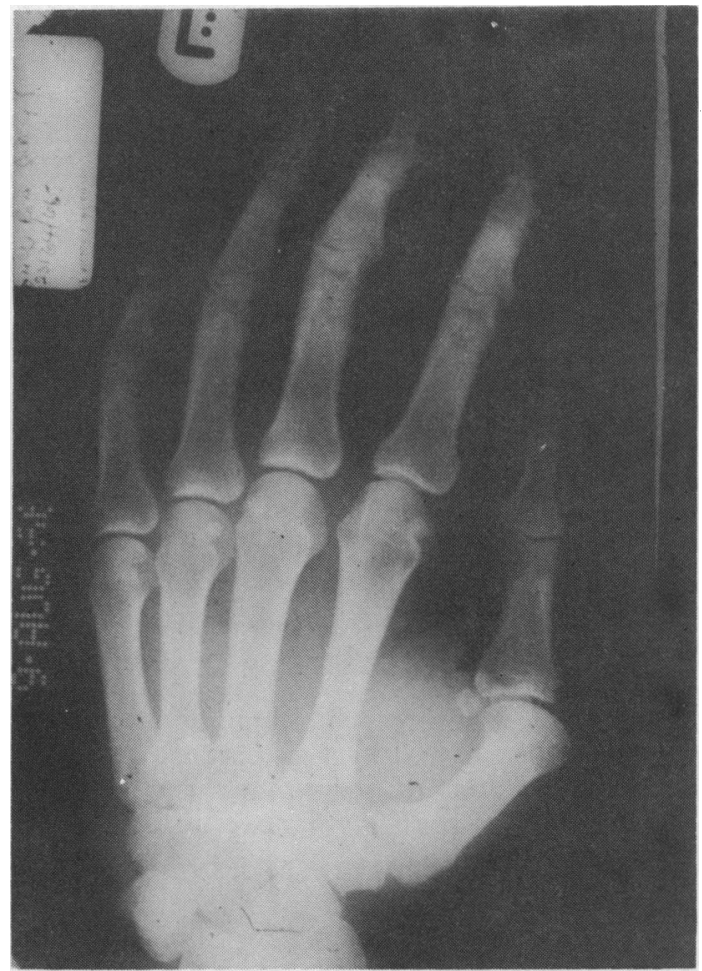

Fig. 8. Arthritic changes at the thumb metacarpophalangeal joint following untreated rupture of the ulnar collateral ligament. out at an early stage when the anatomical changes are still easily identified and corrected. Failure to recognise and adequately treat this injury can lead to arthritic changes in the joint at a later stage. (Fig. 8)

At the proximal end of the thumb metacarpal two types of fracture can occur, the first a transverse fracture of the shaft is of no special significance (Fig. 9) if displaced reduction is carried out and a plaster cast applied to the hand, as for other injuries.

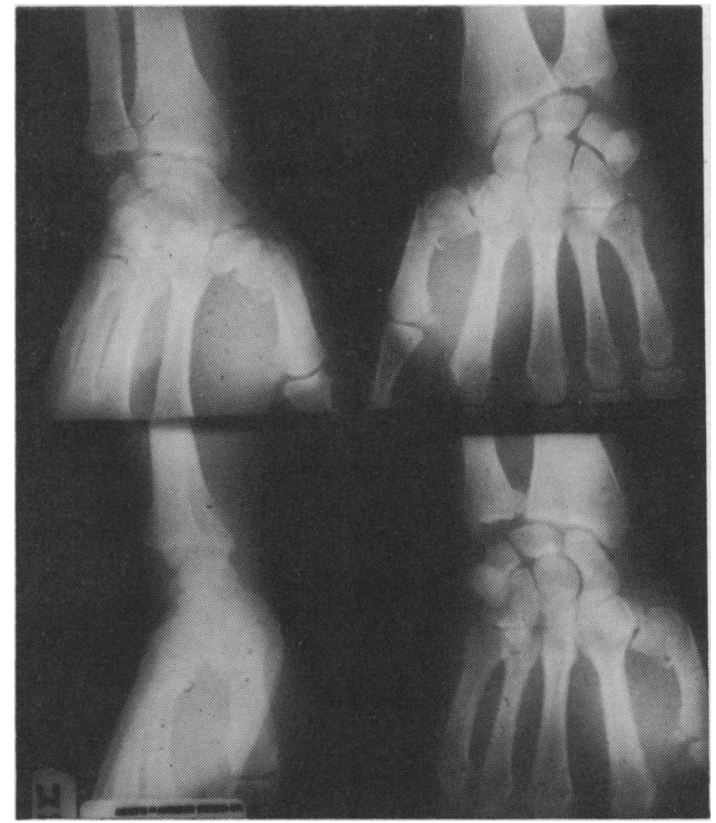

Fig. 9. Transverse fracture of the base of thumb metacarpal.

The second type is of more significance, the Bennett's injury is in fact a fracture dislocation in which the fracture enters the carpo-metacarpal joint and is associated in many cases with considerable displacement. (Fig. 10)

The displacement must be reduced accurately, either by skin or skeletal traction which is incorporated in a plaster cast, if this is not successful, open reduction and fixation by the screw or metal pin should be carried out.

Injuries to the wrist are not common in boxing unless a boxer falls in the ring in which case the injury differs in no way from the common sprains and fractures seen in any casualty department.

Finally in the youth with actively growing epiphyses every effort must be made to protect the hands from too much trauma in the gym, the use of heavy punchbags should be limited, proper gloves and training mitts must be provided and when they are worn they must be 


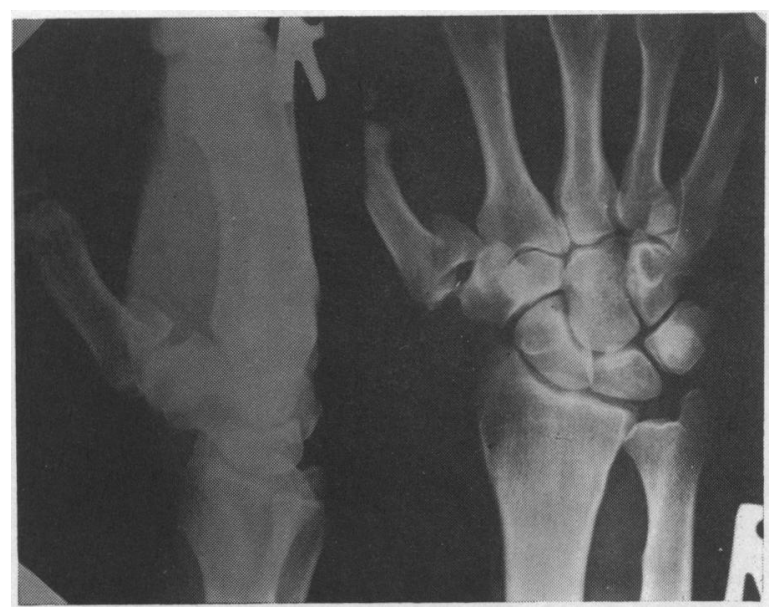

Fig. 10. Bennett's fracture dislocation of thumb metacarpal.

replaced; damage to actively growing epiphyses can

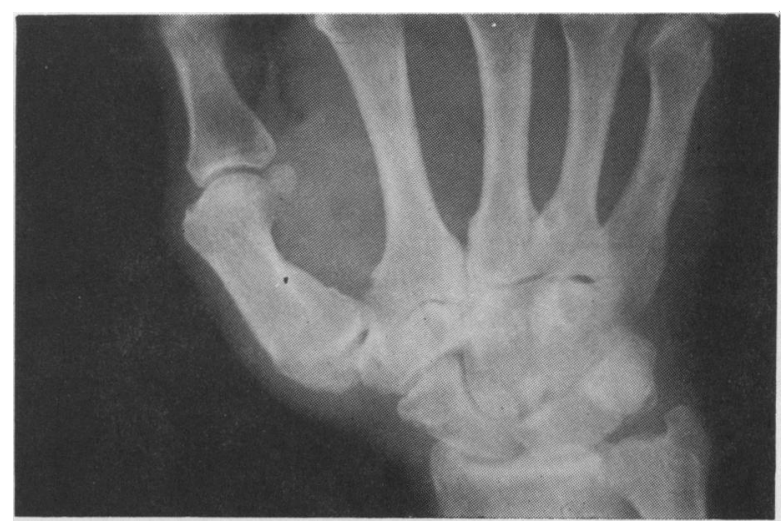

Fig. 11. Old untreated Bennett's fracture.

result in arthritic changes later on, this applies to all sports and it behoves all those responsible for youth training centres to ensure that all precautions are taken to avoid injuries at an early age.

\title{
BOOK REVIEW
}

The A.B.A. Coaching Manual. 'BOXING' by David James.

\author{
Price $£ 2.10 \quad$ Published by Kaye and Ward, London
}

Although boxing is not taught in all schools, it is still a very popular sport amongst youngsters, and is frequently a Youth Club activity. There is therefore a very real need for a good coaching manual which covers not only the technical aspects of the sport, but also gives background information about methods of coaching, and also about the fitness aspect of boxing. All too often tradition has dictated methods of training, and it is high time that modern methods of training were presented to coaches and boxers alike.

This book, which is excellently produced with many good photographs and line drawings meets these needs. The introductory chapter deals with the educational aspects of coaching, and could well prove required reading for student teachers and coaches in any sport. In subsequent chapters the principles of fitness training, interval training circuit and weight training are covered in some detail, and the results of recent research are discussed. Perhaps too much emphasis is placed on high quality interval training, and the need for the development of basic cardio-respiratory fitness by steady state work is not stressed sufficiently; however the tradition of road work for fitness training for boxing will probably redress the balance in practice.

Chapter 7 deals with the medical care of the amateur boxer, and stresses the importance of an initial medical examination - other sports please notice - unfortunately this rule is not infrequently broken at local level. It was perhaps a pity that the writer of this chapter felt it necessary to justify amateur boxing by quoting casualty statistics for various sports. In a coaching manual it would seem inappropriate to justify the sport.

Inevitably in a work to which various authors contribute there will be some divergence of opinions, however it is a very great pity that the chapter of planning the training programme is full of generalisations such as 'use of weights and power circuits', and confuses interval work in the ring with interval training. It would seem that this contributor had not had the opportunity of reading the previous chapters, since his advice is somewhat at variance with the information given earlier.

All in all this is an excellent manual, which could be studied by anyone interested in coaching in general. The technical information is well and clearly presented, and the tactical aspect of the use of the various punches is covered in some detail. All in all this is a book which can be very strongly recommended.

P. R. Travers. 